\title{
Ultrasound-Enhanced Thrombolysis: EKOS EndoWave Infusion Catheter System
}

\author{
Charles A. Owens, M.D. ${ }^{1}$
}

\section{ABSTRACT}

The purpose of the EKOS EndoWave Infusion Catheter System is to enhance catheter-directed thrombolysis by accelerating the fibrinolytic process with the application of ultrasound. Improving the efficiency of the thrombolytic process reduces the treatment time and total lytic dose delivered, thereby lowering the overall cost of therapy and the risk of an associated bleeding complication.

KEYWORDS: Thrombolysis, ultrasound, catheter, clot, interventional radiology

\begin{abstract}
Objectives: Upon completion of this article, the reader should (1) be familiar with the concepts behind ultrasound enhancement of catheter-directed thrombolysis in the treatment of arterial and venous thrombosis; (2) know the clinical studies supporting the use of ultrasound in catheter-directed thrombolysis; (3) understand the different parts of the EKOS EndoWave System and how each part of the catheter system functions.

Accreditation: Tufts University School of Medicine (TUSM) is accredited by the Accreditation Council for Continuing Medical Education to provide continuing medical education for physicians.

Credit: TUSM designates this educational activity for a maximum of 1 AMA PRA Category 1 Credit $^{\text {TM }}$. Physicians should only claim credit commensurate with the extent of their participation in the activity.
\end{abstract}

The use of thrombolytic agents has become routine medical practice in the treatment of embolic and thrombotic vascular occlusions. Thrombolytic therapy has been established as an important therapeutic tool in the treatment of native peripheral artery occlusive disease and thrombosed arterial bypass grafts. The therapeutic success in the treatment of stroke and myocardial infarction relies greatly on the speed the thrombolytic agent can reestablish flow within the affected cerebral or coronary circulation.

In the area of venous thromboembolic disease, catheter-directed thrombolysis with or without assisted mechanical thrombolysis is becoming the standard of medical care in the treatment of acute and subacute deep vein thrombosis (DVT). Anticoagulation therapy is ineffective at removing thrombus of the deep venous system, and when extensive, DVT carries a high risk of developing post-thrombotic syndrome (PTS) and venous status ulcers. ${ }^{1}$ To avoid the development of PTS, aggressive therapy to remove the venous thrombus/ venous obstruction must be undertaken before irreversible valvular damage occurs. ${ }^{2}$

The EKOS EndoWave Infusion Catheter System (EKOS Corporation, Bothell, WA) enhances catheterdirected thrombolysis by accelerating the fibrinolytic process via the application of ultrasound. Improving the efficiency of the thrombolytic process reduces the treatment time and total lytic dose delivered. The overall cost of therapy and the risk of an associated bleeding complication thus are reduced.
${ }^{1}$ Division of Interventional Radiology, University of Illinois Medical Center, Chicago, Illinois.

Address for correspondence and reprint requests: Charles A. Owens, M.D., Division of Interventional Radiology (M/C 931), University of Illinois Medical Center, 1740 West Taylor Street, Chicago, IL 60612 (e-mail: cowens@uic.edu).
New Tools and Techniques in Interventional Radiology; Guest Editor, Martin G. Radvany, M.D.

Semin Intervent Radiol 2008;25:37-41. Copyright (C) 2008 by Thieme Medical Publishers, Inc., 333 Seventh Avenue, New York, NY 10001, USA. Tel: +1(212) 584-4662.

DOI 10.1055/s-2008-1052304. ISSN 0739-9529. 


\section{ULTRASOUND-ENHANCED THROMBOLYSIS}

One of the most important determinants affecting the rate of fibrinolysis is the speed the thrombolytic agent can be delivered to the plasminogen receptor sites within the thrombus. ${ }^{3,4}$ Catheter-directed thrombolysis relies on thermal-mediated diffusion, a slow process requiring a high concentration gradient to drive the reagent into the clot. ${ }^{5}$ In 1989, Kudo pioneered the idea of enhancing fibrinolysis by applying ultrasound energy to the thrombolytic process. ${ }^{6}$ Ultrasound when applied to the thrombosed vessel during thrombolysis enhances the thrombolytic process by accelerating the contact of the lytic agent with plasminogen receptor sites within the thrombus. Acoustic microstreamimg created by the ultrasound pulses augments the transportation of the lytic agent, disseminating it throughout the clot. The energy transmitted by the ultrasound waves also separates/loosens the fibrin strands, increasing the surface area of the thrombus and making more plasminogen activator receptor sites available to the lytic agent.

Francis et al demonstrated that when ultrasound was applied to an in vitro model of lysing thrombus, less thrombolytic agent (recombinant tissue-type plasminogen activator [rt-PA]) was required to achieve an equal amount of fibrinolysis and with no evidence of mechanical fragmentation of the clot. ${ }^{7}$ In a similar in vitro study, Suchkova et al demonstrated with the same concentration of thrombolytic agent $(1 \mu \mathrm{g} / \mathrm{mL} \mathrm{t}-\mathrm{PA})$, the amount of fibrinolysis could be greatly enhanced by the application of low-intensity $(40-\mathrm{kHz})$ ultrasound waves. ${ }^{8}$ Transcutaneous application of ultrasound waves to enhance fibrinolysis also proved effective in animal studies. Using a rabbit model of arterial thrombosis, Riggs et al demonstrated a several fold increase in thrombolysis by combining ultrasound with streptokinase. ${ }^{9}$ Luo et al achieved faster thrombolysis in a rabbit model of arterial thrombosis by combining transcutaneous ultrasound with thrombolytic therapy. ${ }^{10}$

Using a prototype ultrasound wire transducer attached to a catheter, Tachibana et al were able to demonstrate enhanced thrombolysis with urokinase. ${ }^{11,12}$ Shlansky-Goldberg at el demonstrated acceleration of urokinase-mediated thrombolysis of whole blood clots using a $9 \mathrm{~F}$ catheter and a piezoelectric crystal $(640 \mathrm{kHz})$ placed at the end of the catheter. ${ }^{13}$ Atar et al demonstrated enhanced arterial thrombolysis in nine canines using a transducer-tipped high-frequency ultrasound drug-delivery catheter. ${ }^{14}$

In 2004, the EKOS Corporation gained approval from the Food and Drug Administration to manufacture and distribute the EKOS EndoWave System for the "controlled and selective infusion of physician-specified fluids, including thrombolytics, into the peripheral vasculature" (Figs. 1 and 2). A similar but smaller device, the EKOS Micro Infusion Catheter, is being investi- gated for the purpose of accelerating catheter-directed thrombolysis of acute embolic strokes. ${ }^{15}$

\section{THE EKOS ENDOWAVE SYSTEM}

The EndoWave System, manufactured by EKOS Corporation, consists of a $5.2 \mathrm{~F}, 106-\mathrm{cm}-\mathrm{long}$ infusion catheter, an ultrasound core wire, and a control unit with catheter interface cables. A series of laser-cut microinfusion pores are located along the treatment segment of the catheter for the delivery of the thrombolytic agent. The microinfusion pores are positioned to optimize interface with corresponding ultrasound transducers. The treatment segment on each catheter varies from $6 \mathrm{~cm}$ to $50 \mathrm{~cm}$ in length.

The infusion catheter is guided over a standard 0.035 -inch wire and positioned within the thrombus. The guidewire is then replaced with a matching ultrasound core wire that fits into the center lumen of the catheter (Fig. 1). The wire consists of miniaturized ultrasound transducers, spaced $1 \mathrm{~cm}$ apart, that deliver pulsed high-frequency (2-MHz), low-power ultrasound waves (Figs. 3 and 4). Normal saline is infused via a coolant port connected to the central lumen and is used to diffuse heat energy generated by the ultrasound transducer. Both the infusion catheter and the ultrasound core wire are attached to a catheter interface cable, which in turn is connected to the system control unit (Fig. 2).

The control unit automatically adjusts the electrical input delivered to the ultrasound transducers, optimizing the emitted level of power. A series of thermocouples is positioned along the catheter, which transmit local temperature back to the control unit. Temperature changes perceived by the thermocouples allow the control unit to alter the power level of the ultrasound transducers. Power fluctuations are

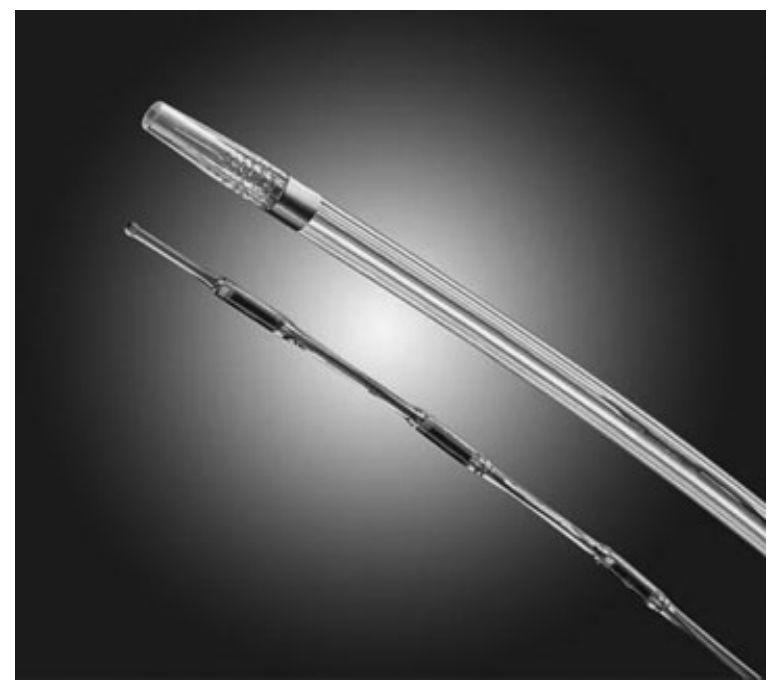

Figure 1 The EKOS EndoWave ultrasound core wire and multilumen infusion catheter. Note three ultrasound transducers are seen on the wire. 


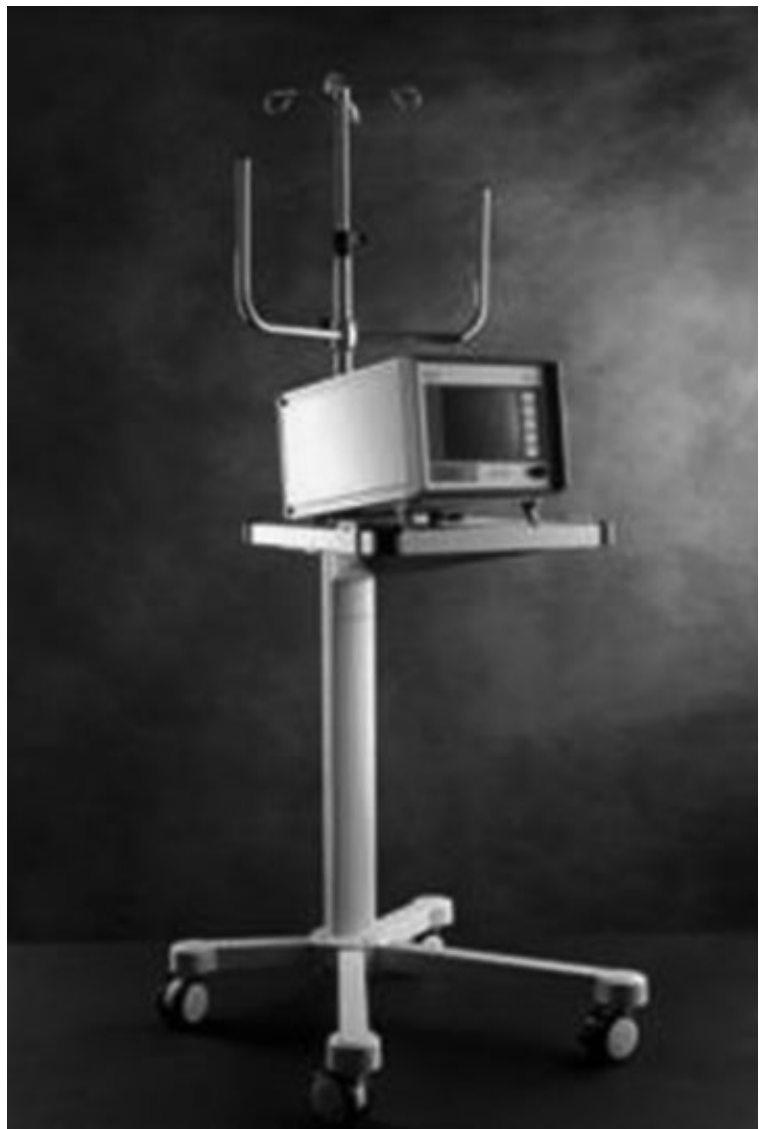

Figure 2 The EKOS Control Unit adjusts power input to the ultrasound transducers.

graphically displayed on the control unit, which may be used to identify when blood flow has been reestablished and thus signaling when angiographic reassessment should be performed.

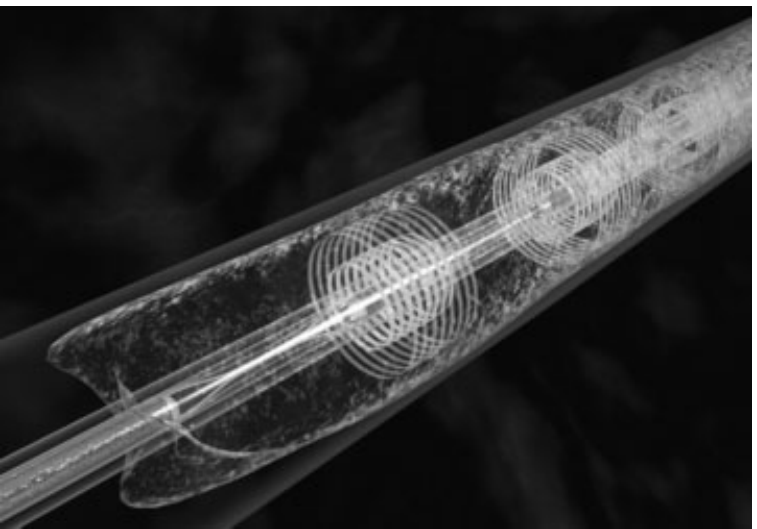

Figure 3 Graphic illustration of ultrasound emitted from three transducers along the core wire.

\section{SUPPORTING CLINICAL DATA}

Published reports have described clinical success using ultrasound-enhanced thrombolysis in the treatment of peripheral arterial occlusive disease. McNamara et al reported using the EKOS EndoWave System on 54 peripheral arterial occlusions in 51 patients $^{16}$ (Fig. 5). Complete lysis was achieved in 46 occlusions (85.2\%); only partial lysis was achieved in 4 (7.4\%) and no lysis in 4 (7.4\%). The average time of infusion was 17.3 hours, and one bleeding complication was reported (1.9\%). In another study, Wissgott et al reported the treatment of 20 peripheral artery occlusions in 19 patients using the EKOS EndoWave System $^{17}$ (Fig. 6). In their study, complete lysis was achieved in 19 (95\%) patients and partial lysis in 1 patient (5\%). The average time of infusion was 16.8 hours ( \pm 11.1 hours). They reported no complications. The multicenter Thrombolysis Or
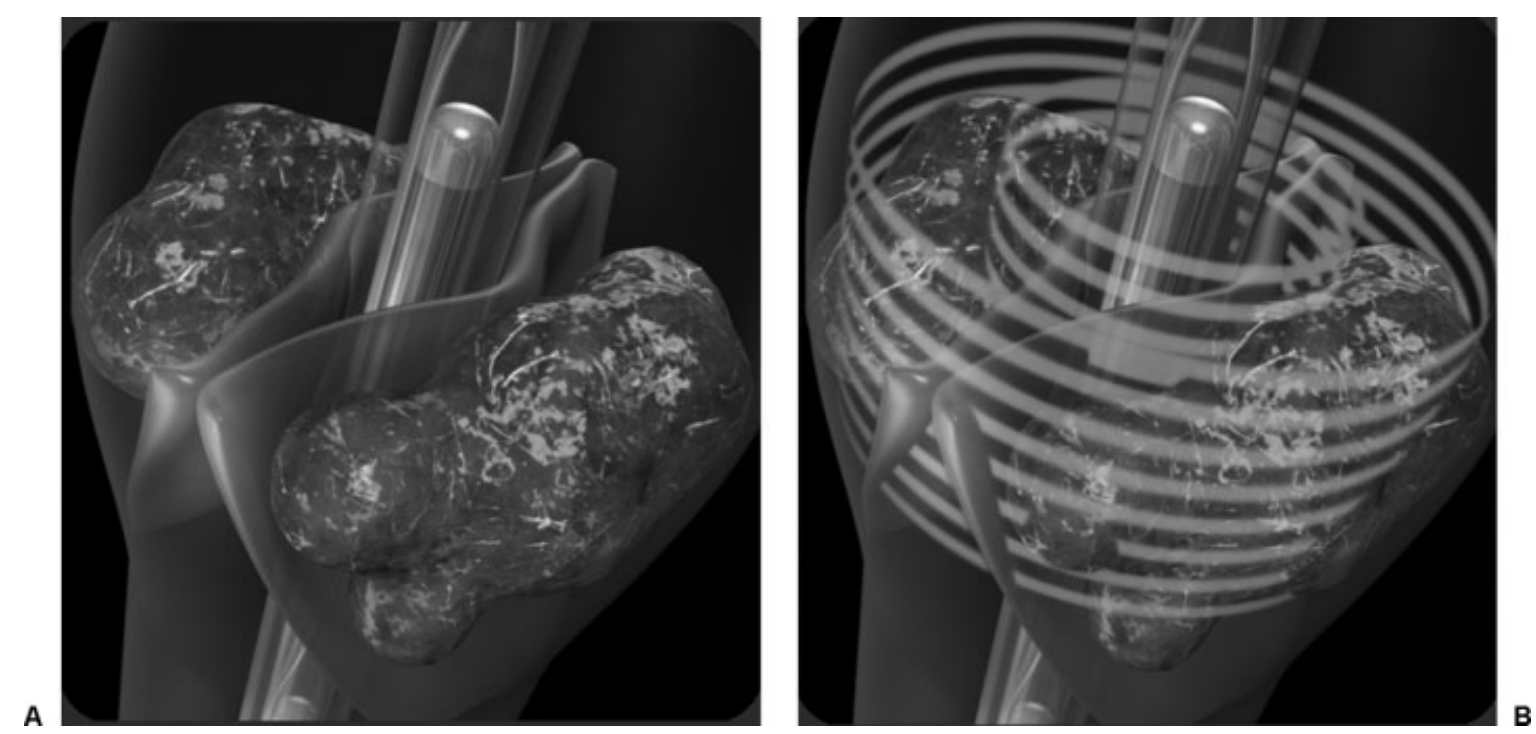

Figure 4 (A) Graphic illustration of the EKOS ultrasound core wire and infusion catheter positioned within a thrombosed vein. (B) Graphic illustration of the ultrasound waves penetrating the venous valves, impacting thrombus located behind them. 


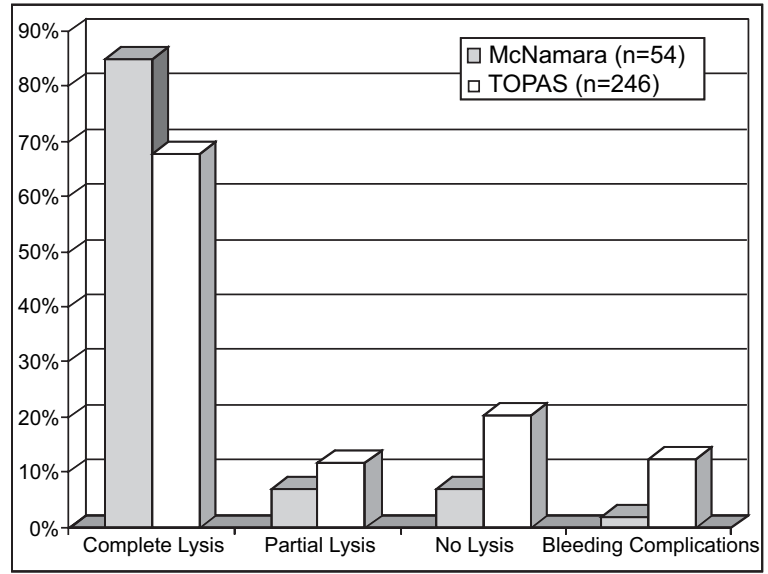

Figure 5 Fifty-four arterial occlusions treated with thrombolytics and the EKOS EndoWave system (solid gray bars) are compared with the Thrombolysis Or Peripheral Arterial Surgery (TOPAS) study (striped bars). Note the $85 \%$ complete lysis and $2 \%$ major bleeding complications with ultrasound-enhanced thrombolysis compared with 68\% complete lysis and $12 \%$ major bleeding complications in the TOPAS study.

Peripheral Arterial Surgery (TOPAS) study was used as a historical control in both studies. ${ }^{18}$ In the TOPAS trial, follow-up angiograms were performed in 246 patients with arterial occlusions treated with urokinase only. They reported complete lysis in 167 patients $(67.9 \%)$, partial lysis in $20(11.8 \%)$, and failed lysis in 50 (20.3\%). The average time of thrombolytic infusion was $24.4 \pm 0.86$ hours with a reported major bleeding complication rate of $12.5 \%$.

Assessing the usefulness of ultrasound-enhanced thrombolysis in venous disease, Raabe reported the

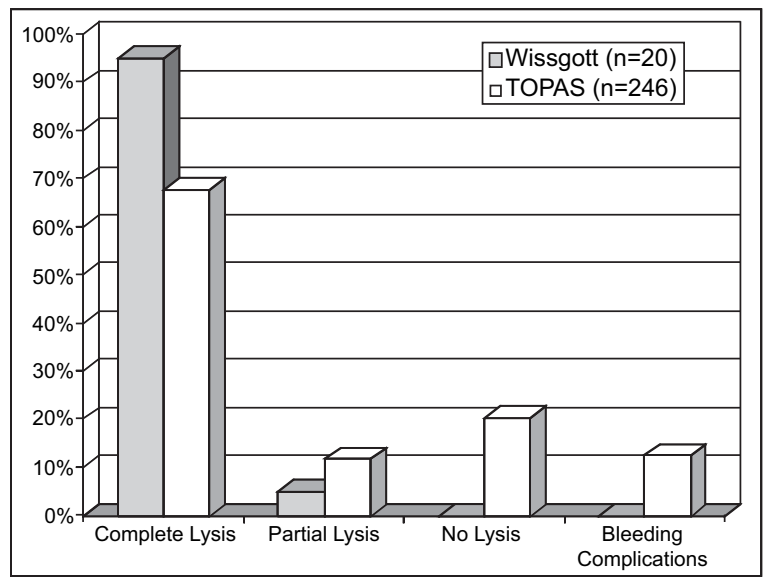

Figure 6 Twenty arterial occlusions treated with thrombolytics and the EKOS EndoWave system (solid gray bars) are compared with the Thrombolysis Or Peripheral Arterial Surgery (TOPAS) study (striped bars). Note the 95\% complete lysis and no major bleeding complications with ultrasoundenhanced thrombolysis compared with $68 \%$ complete lysis and $12 \%$ major bleeding complications in the TOPAS study.

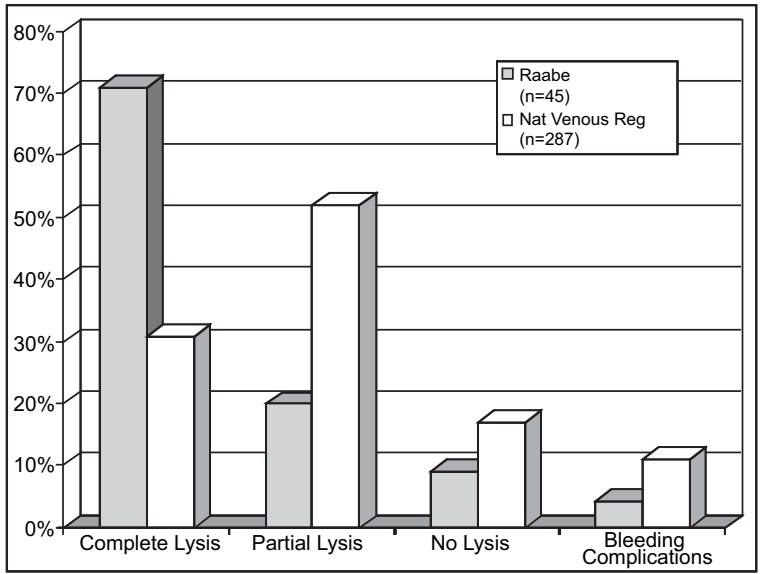

Figure 7 Forty-five venous occlusions treated with thrombolytics and the EKOS EndoWave system (solid gray bars) are compared with the National Venous Thrombosis Registry (striped bars). Note the $71 \%$ complete lysis and $4.4 \%$ major bleeding complications with ultrasound-enhanced thrombolysis compared with $31 \%$ complete lysis and $11 \%$ major bleeding complications in the National Venous Thrombosis Registry.

treatment of 45 venous occlusions (25 lower extremity, 17 upper extremity, 2 hepatic vein, 1 arterial venous fistula) using the EKOS EndoWave System ${ }^{19}$ (Fig. 7). Venous lysis was performed using reteplase (47\%), urokinase (24\%), alteplase (16\%), and tenecteplase (13\%). Complete lysis was achieved in $71 \%$ and partial lysis in an additional $20 \%$. The average time to achieve complete lysis was 24.7 hours. Two bleeding complications (4.4\%) were reported. Raabe compared his results with those reported from the National Venous Thrombosis Registry, a prospective multicenter study that assessed the treatment of 221 iliofemoral and 79 femoropopliteal venous occlusions with catheter-directed thrombolysis therapy in 303 limbs of 287 patients. $^{20}$ The National Venous Thrombosis Registry reported complete lysis in 96 patients (31\%), partial lysis in 162 $(52 \%)$, and no lysis in 54 (17\%) with an average lysis time of 53.4 hours and a major bleeding complication rate of $11 \%$.

In another study, Motarjeme reported improvement in lysis time and success of catheter-directed thrombolysis using the EndoWave System in the treatment of 36 vascular occlusions (24 arterial and 12 venous) in 33 patients. ${ }^{21}$ Complete thrombolysis was reported in $96 \%$ of arterial occlusions and $83 \%$ of venous occlusions. Two thrombolysis failures were reported in the study: one chronic superficial femoral artery occlusion and one chronic iliac vein occlusion. The average time to achieve complete thrombolysis was 16.4 hours ( 3 to 25 hours) for arterial occlusions and 21.2 hours (6 to 43 hours) for venous occlusions. No device failures and no associated complications were reported. 


\section{CONCLUSIONS}

The need to obtain faster and more efficient fibrinolysis is self-evident. In cases of coronary or cerebral thrombosis, expediency to restore blood flow lowers patient morbidity and mortality. In the area of acute and subacute peripheral artery occlusions, more efficient thrombolysis should result in greater limb salvage with fewer associated bleeding complications. In the treatment of venous thromboembolic disease, faster thrombolysis should translate into shorter treatment times requiring less thrombolytic agent and resulting in cost reduction and a decreased incidence of PTS.

Currently no publications have addressed the cost effectiveness of using the EKOS EndoWave Infusion Catheter System during arterial or venous thrombolysis. The initial investment in purchasing the control unit is $\$ 14,500$. Each infusion catheter with ultrasound wire and interface cable retails for $\$ 2295$. If use of the EKOS EndoWave Infusion Catheter System can shorten the time required for catheter-directed thrombolysis, it should translate into a substantial cost savings by decreasing the number of days required for monitoring in the intensive care unit, the amount of thrombolytic medication, and the number of laboratory tests and angiograms. Furthermore, the cost savings achieved by lowering the complication rate may also prove to be significant.

Preclinical in vitro and in vivo research supports the safety and efficiency of adding ultrasound to chemical thrombolysis. ${ }^{6-14}$ Clinical studies to date demonstrated significant improvement in the treatment of venous and arterial thrombosis using ultrasound-enhanced thrombolysis with the EKOS EndoWave System. ${ }^{16,17,19,21}$ Although small population sizes were used (20 to 54 patients per study), each clinical study reported higher success rates with shorter treatment times and lower complication rates when compared with historical controls. Larger multicenter controlled studies are needed, however, to confirm these initial clinical results and to better define the overall best endovascular therapy to use in the treatment of arterial and venous occlusions.

\section{REFERENCES}

1. Strandness DE, Langlois $\mathrm{Y}$, Cramer M, et al. Long-term sequelae of acute venous thrombosis. JAMA 1983;250:12891292

2. Meissner MH, Manzo RA, Bergelin RO, et al. Deep vein insufficiency: the relationship between lysis and subsequent reflux. J Vasc Surg 1993;18:596-605
3. Diamond SL, Anand S. Inner clot diffusion and permeation during fibrinolysis. Biophys J 1993;65:2622-2643

4. Francis CW, Blinc A, Lee S, et al. Ultrasound accelerates transport of recombinant tissue plasminogen activator into clots. Ultrasound Med Biol 1995;21:419-424

5. Francis CW. Ultrasound-enhanced thrombolysis. Echocardiography 2001;18:239-246

6. Kudo S. Thrombolysis with ultrasound effect. Tokyo Jikeikai Med J 1989;104:1005-1012

7. Francis CW, Onundarson PT, Carstensen EL, et al. Enhancement of fibrinolysis in vitro by ultrasound. J Clin Invest 1992;90:2063-2068

8. Suchkova V, Siddiqi FN, Carstensen EL, et al. Enhancement of fibrinolysis with $40 \mathrm{kHz}$ ultrasound. Circulation 1998; 98:1030-1035

9. Riggs PN, Francis CW, Bartos SR, et al. Ultrasound enhancement of rabbit femoral artery thrombolysis. Cardiovasc Surg 1997;5:201-207

10. Luo H, Nishioka T, Fishbein MC, et al. Transcutaneous ultrasound augments lysis of arterial thrombi in vivo. Circulation 1996;94:775-778

11. Tachibana K. Enhancement of fibrinolysis with ultrasound energy. J Vasc Interv Radiol 1992;3:299-303

12. Tachibana K, Tachibana S. Prototype therapeutic ultrasound emitting catheter for accelerating thrombolysis. J Ultrasound Med 1997;16:529-535

13. Shlansky-Goldberg RD, Cines DB, Sehgal CM. Catheterdelivered ultrasound potentiates in vitro thrombolysis. J Vasc Interv Radiol 1996;7:313-320

14. Atar S, Luo H, Nagai T, et al. Arterial thrombus dissolution in vivo using a transducer-tipped, high-frequency ultrasound catheter and local low-dose urokinase delivery. J Endovasc Ther 2001;8:282-290

15. Mahon BR, Nesbit GM, Barnwell SL, et al. North American clinical experience with the EKOS MicroLysUS infusion catheter for the treatment of embolic stroke. AJNR Am J Neuroradiol 2003;24:534-538

16. McNamara T, Parikh S, Motarjeme Aet al. Ultrasound accelerated thrombolysis in peripheral arterial occlusion: complete lysis rates using the EKOS Lysus system in the same day setting. Presented at: Transcatheter Cardiovascular Therapeutics; October 2005; Abstract 365

17. Wissgott C, Richter A, Kamusella P, Steinkamp HJ. Treatment of critical limb ischemia using ultrasound-enhanced thrombolysis (PARES Trial): final results. J Endovasc Ther 2007;14:438-443

18. Ouriel K, Veith FJ, Sasahara AA. A comparison of recombinant urokinase with vascular surgery as initial treatment for acute arterial occlusion of the legs. N Engl J Med 1998;338:1105-1111

19. Raabe RA. Ultrasound-facilitated thrombolysis in treating DVT. Endovascular Today 2006;5:75-79

20. Mewissen MW, Seabrook GR, Meissner MH, et al. Catheter-directed thrombolysis for lower extremity deep venous thrombosis: report of a national multicenter registry. Radiology 1999;211:39-49

21. Motarjeme A. Ultrasound-enhanced thrombolysis. J Endovasc Ther 2007;14:251-256 